\title{
EL DESARROLLO DEL TALENTO SOBRESALIENTE EN LOS ESTUDIANTES ADOLESCENTES
}

Incontables son las maravillas del mundo, pero ninguna más maravillosa que el hombre.

Sófocles (469-406 A.C.).

Vivian Ma. Pacheco Urbina

\section{Introducción}

La época actual es una de las más interesantes, debido a la cantidad de cambios que se suscitan con gran rapidez en todos los campos del saber, cambios que producen re-

Resumen: El extraordinario potencial del ser humano, que se manifiesta en el talento sobresaliente, en las aptitudes y las habilidades de algunos estudiantes, denota en su personalidad y en el excelente rendimiento académico que obtienen, y en su participación activa en el proceso educativo; se proyecta asi mismo en su interés por los avances científicos y tecnológicos; en la valiosa producción intelectual, que se traduce en teorias, investigaciones u obras literarias; en la riqueza espiritual que se expresa en su relación con la familia y con la sociedad; en su creación artística, que se expresa en la pintura, la escultura, la música y la poesía.

En el contexto de los factores que intervienen en la excelencia académica de estudiantes de primeros promedios de admisión de la U.C.R., el presente artículo analiza las concepciones y lineas de pensamiento de autores que sustentan teorias sobre el desarrollo del talento en el adolescente: Freeman (1992), Benito (1990), Maker (1995), Renzulli (1981): "La teoría de los tres anillos" y Mönks y W. van Boxtel (1992): "Modelo Triádico de la sobredotación".

Se plantea asimismo, las necesidades educativas y características del desarrollo del adolescente talentoso en el núcleo familiar y en los marcos sociales en los que establece una interacción dinámica con otras personas significativas para el desarrollo de sus capacidades cognitivas. tos de actualización, innovación y creatividad en el ser humano.

Para asumir esos retos, Pöggeler señala que:

el derecho a la educación, se trata del derecho al conocimiento y al desarrollo de aptitudes e intereses, facultades e inclinaciones, algo a alcanzar por medio de la educación de la persona (Pöggeler, 1989, p. 32).

Así, la educación prevalece a través de la historia, como un derecho fundamental que tiene el ser humano, independiente en su totalidad del género, edad, credo, cultura y sistema político.

Por su parte, Machado al exponer sus ideas en torno a la educación, señala:

La primera necesidad humana es la educación. Por eso, en la vida social lo fundamental es aprender. El hombre nace con la capacidad de aprenderlo todo, pero sin saber nada.

Todo lo que realiza, en algún momento lo ha aprendido. Y puede aprender a desarrollar un sinfín de potencialidades.

Para vivir, tenemos que aprender a vivir (Machado, 1983, p. 144).

Este derecho fomenta en el individuo el desarrollo integral y armónico de su intelecto, 
personalidad, aptitudes, creatividad y facultades. A través de la educación en el hombre y la mujer, se forman valores morales, religiosos, cívicos y culturales; se desarrollan actitudes, habilidades, destrezas y se adquieren conocimientos teóricos y prácticos fundamentales para el desarrollo pleno del potencial humano.

Este proceso de formación, se adquiere en el núcleo familiar, en el sistema de enseñanza al cual se tiene acceso y en el contexto sociocultural que rodea al individuo.

Al respecto, Piaget indica:

La educación, para la mayoría de las personas, significa guiar al niño hacia la meta de semejarse al adulto típico de la sociedad en que vive. Para mí, la educación significa formar creadores, aún si sabemos que su número ha de ser escaso, aún si las creaciones de un determinado individuo podrán ser consideradas limitadas, en comparación con las de otros. Aún así, debemos formar seres con inventiva, espíritu innovador y no conformistas (ref. por García, 1986, p. 1).

La educación se dirige a formar integralmente a las personas, para que asuman con responsabilidad nuevos roles sociales, fundamentales para su participación en esta época, que demandan un ser humano con una conciencia analítica y crítica de las necesidades de su cultura actual; capaz de afrontar los retos científicos, tecnológicos y productivos que ocurren en la sociedad contemporánea.

Al respecto, Durkheim define la educación como:

el medio por el cual logrará crear en el corazón de las jóvenes generaciones, las condiciones esenciales para la propia existencia ... la acción ejercida por las generaciones adultas sobre las que no están todavía maduras para la vida social, tiene como objetivo suscitar y desarrollar en el niño cierto número de estados físicos, intelectuales y morales, que requieren en él, tanto la sociedad política en su conjunto, como el ambiente particular al que está destinado de manera específica (ref. por López, 1994, p. 39-40).

Las profundas transformaciones que enfrenta la sociedad y que repercuten en todos los sectores de la población, generan interrogantes en torno a la atención que la educación ofrece al desarrollo del talento cognoscitivo, a las aptitudes, habilidades, creatividad y valores del individuo, ante un entorno cambiante que le demanda participación activa, análisis y reflexión de su papel en la sociedad del futuro.

Al respecto, Fonseca plantea:

Es evidente que para desarrollar el talento tenemos que centrar nuestra mirada en el individuo. Pero no podemos perder jamás de vista, el entorno social y el papel que el talento de una persona puede tener en el bienestar del conjunto. Aún cuando el talento tenga un sentido individual de potenciación personal, éste debe convertirse, en toda su plenitud, en un bien social (Fonseca, 1991, p. 29).

En este proceso, adquiere relevancia las alternativas que la educación ofrece para el desarrollo del talento, las habilidades y aptitudes que tiene cada persona, ya que es a través de ésta, que se le posibilita ascender en la escala social y, además, obtener un nivel de vida más satisfactorio para él y su familia.

En el presente artículo, se considera de talento sobresaliente, a aquella persona cuyo rendimiento sea superior al de la generalidad, en cualquier área de la conducta humana socialmente valiosa, en el campo intelectual, artístico, cultural y de relaciones humanas.

\section{El talento sobresaliente en la adolescencia}

Desde la infancia, el estudiante talentoso presenta rasgos o características que lo diferencian del resto del grupo escolar, por lo que programas, currículos y métodos de enseñanza deben contribuir al desarrollo pleno de su capacidad, enfatizando en áreas de suma importancia en la actualidad, a saber:

capacidad intelectual general, capacidades específicas, creatividad, realización demostrada y potencial excepcional... (Porto, ref. por Benito, 1990, p. 88). 
Al considerar las características de los estudiantes talentosos y las necesidades particulares que éstos presentan, se debe enfatizar en las previsiones educativas específicas que requieren de los sistemas educativos.

Freeman plantea:

Para que se pueda poner en práctica cualquier tipo de educación para los superdotados, es esencial conocer en qué medida estos niños difieren en sus necesidades educativas respecto de otros. Estas diferencias pueden ser resumidas en forma breve: cualquiera que sea su talento, aquellos que son excepcionalmente capaces de aprender lo harán con mayor rapidez, profundidad y extensión que otros niños (Freeman, 1992, p. 32).

Las necesidades educativas de los estudiantes talentosos sugieren modificaciones específicas en los programas de estudio, que les permitan el desarrollo de su capacidad cognitiva, habilidades y destrezas. Existen desde hace varios años, ponencias de diversos autores, que proponen la congruencia del programa con las necesidades de estos estudiantes; Gallagher al respecto expone:

Un necesario cambio en el contenido de las áreas tradicionales de aprendizaje, por ejemplo, matemáticas, historia...; la inclusión de nuevas áreas de estudio o de unidades curriculares más complejas; un material más avanzado y la investigación de problemas reales.

La inclusión de destrezas especiales, como la solución de problemas y el uso de métodos de aprendizaje por descubrimiento y métodos creativos.

- Un cambio en el ambiente de aprendizaje que suponga la creación de un clima facilitador, que puede variar desde el contacto especial durante unas horas al día o semana con niños de similar capacidad, a través de escuelas especiales (Porto, ref. por Benito, 1990, p. 89).
Los estudiantes talentosos difieren de sus compañeros en el modo de proceder que asumen ante las necesidades básicas de aprendizaje; destacan como rasgos característicos su automotivación, persistencia e independencia.

En relación con este planteamiento, Feldhusen y Wyman presentan su criterio de las necesidades básicas que deben ser consideradas en los programas de estudio del alumno talentoso.

\section{Necesidades básicas del estudiante}

Máximo logro de destrezas y conceptos básicos.

- $\quad$ Actividades de aprendizaje a nivel y ritmo apropiado.

- Experiencias en pensamiento creativo y solución de problemas.

- Desarrollo de la capacidad convergente, especialmente de la deducción lógica y la solución de problemas.

- Estimulación de la imaginación y las capacidades espaciales.

- Desarrollo de la autoconciencia y aceptación de las capacidades, intereses y necesidades propias.

Estimulación para perseguir metas y aspiraciones de alto nivel.

- Desarrollo de la independencia, autodirección y disciplina en el aprendizaje.

Experiencias de relación con otros estudiantes intelectual, artística y efectivamente muy capacitados, creativos y talentosos.

Amplias fuentes de información sobre diversos temas. 
- Exposición a una variedad de campos de estudio, arte, profesiones y ocupación.

- $\quad$ Acceso y estimulación para la lectura. (Porto, ref. por Benito, 1990, p. 89-90).

$\mathrm{Al}$ reconocer las diferencias individuales del estudiante talentoso, el centro educativo, el personal administrativo y los profesores deben promover un ambiente flexible, en el que se acepte su potencialidad, la valoración de su progreso y su trabajo personal, a la vez que se fomente la cooperación entre los estudiantes y su interrelación social.

Al respecto, Porto indica:

El rol del profesor en este contexto, es también diferente. Debe otorgar gradualmente la responsabilidad del aprendizaje al alumno, para que llegue a ser un aprendiz independiente. Debe, además, ser la fuente primaria que ha de presentar múltiples alternativas y opciones, capaces de generar en el alumno una buena motivación y la toma de decisiones y responsabilidades. Su actuación debe ser flexible, modificable y ajustada a las diferencias y necesidades individuales, promoviendo el desarrollo de la creatividad, originalidad y divergencia, guiando y orientando el alumno en su trabajo (Porto, ref. por Benito, 1990, p. 102).

El ambiente facilitador, al promover el trabajo en grupo y el fortalecimiento de las relaciones entre los alumnos, contribuye a que el estudiante tome decisiones y analice el avance de su trabajo. Es importante que el estudiante talentoso asuma responsabilidades y acepte las aportaciones de los demás con igual criterio de validez.

En su análisis sobre el ambiente de aprendizaje, Maker (1995) indica que éste se refiere al contexto en el cual se desarrolla, considerando tanto el contexto físico de la escuela y del aula, como el clima psicológico de la clase.

El autor recomienda modificaciones para el ambiente escolar, basadas en tres condiciones:

Que sean preferidas por los superdotados como grupo.
Que sean necesarias para implementar las modificaciones de contenidos, procesos y productos.

- Que se efectúen de acuerdo con las características de los estudiantes superdotados.

Las modificaciones propuestas por Maker son las siguientes:

- $\quad$ El ambiente de aprendizaje debería estar centrado en el estudiante más que en el maestro.

- Los maestros deberían alentar la independencia más que la dependencia y los estudiantes asumir la responsabilidad de solucionar sus propios problemas, aun aquellos relacionados con el manejo de la clase.

- $\quad$ El ambiente debe ser abierto más que cerrado.

- $\quad$ Las reacciones de los maestros ante las ideas y productos de los estudiantes deben ser de aceptación más que de juicio.

- El ambiente físico y psicológico debe ser complejo más que simple.

- El ambiente de aprendizaje debería permitir una gran movilidad más que movimientos restringidos. (Maker, 1995, p. 149).

De acuerdo con las investigaciones realizadas en este campo, estos cambios permiten implementar un currículo adecuado a las necesidades y expectativas de los estudiantes talentosos. Estas modificaciones requieren para su aplicación en el currículo escolar, de un análisis de las características de cada sistema educativo en particular; considerando, entre otros recursos, los humanos, los físicos, los económicos, las características 
del estudiante talentoso, las metas, los objetivos, los programas, los procesos, las estrategias y los productos educativos.

En sus investigaciones, Gallagher y sus colegas identificaron siete estrategias administrativas principales, que se utilizan en la actualidad en los programas para superdotados:

1. Enriquecimiento en la clase. Provisión de un programa diferenciado de estudio para el superdotado, por el maestro de grado, dentro de la clase común, sin la ayuda de un recurso externo o de un maestro consultor.

2. Programa con maestro consultor. Instrucción diferenciada provista dentro de la clase regular por el maestro de grado, con la asistencia de un maestro consultor especialmente entrenado.

3. Cuarto de recursos/programa de extracción. Los estudiantes superdotados dejan la clase común para recibir instrucción diferenciada impartida por un maestro especialmente entrenado.

4. Programa con un mentor comunitario. Los estudiantes superdotados interactúan individualmente con miembros seleccionados de la comunidad, por un extenso período y analizan un tema de interés especial para el niño.

5. Programa de estudios independiente. La instrucción diferenciada consiste en un proyecto de estudio independiente, supervisado por un adulto calificado.

6. Clase especial. Los estudiantes superdotados se agrupan juntos y reciben instrucción de un maestro especialmente entrenado.

7. Escuela especial. Los estudiantes superdotados reciben instrucción diferenciada en una escuela especial establecida con ese propósito. (Ref. por Maker, 1995, p. 141).
Sobre estos programas, Maker (1995) indica que el de mayor aceptación por los directores estatales de programas para niños superdotados, los padres y los profesores, es el de recursos para el nivel elemental y las clases de avanzados en la secundaria.

Destaca la importancia de considerar, para diseñar un programa, las características de los alumnos, el contexto y el clima escolar, las destrezas y las actitudes de los demás miembros del personal escolar, los valores de la comunidad y los resultados deseables para los niños.

De la riqueza del aporte de experiencias que el estudiante talentoso adquiere del núcleo familiar, del ambiente de aprendizaje y del contexto social que le circunda, obtiene una serie de elementos que propician el desarrollo de sus potencialidades.

\section{Los adolescentes en el contexto de la sobredotación}

En el proceso del desarrollo del individuo, la adolescencia es una etapa en la vida en la que se dan una serie de transformaciones biológicas y cognitivas, que ocurren entre la pubertad y la madurez.

En este período emergen una gran variedad de cambios conductuales y se da una gran diferenciación de las capacidades cognitivas, el proceso de reflexión es más profundo, el pensamiento es crítico y analítico; se experimentan cambios en sus relaciones con el núcleo familiar y con el grupo social en el que se desenvuelve.

La concepción de la sobredotación de los "Tres Anillos" de Renzulli (ref. por Mönks y W. van Boxtel, 1992), que implica el factor cognitivo y otros factores, destaca la combinación entrelazada de tres grupos de rasgos: capacidad por encima de lo normal, compromiso con la tarea y creatividad.

Renzulli y colaboradores, con base en los resultados de una amplia investigación empírica, describen la sobredotación como: 
Una interacción entre tres grupos básicos de rasgos humanos; estos grupos son capacidades generales por encima de la media, altos niveles de implicación en la tarea y altos niveles de creatividad. Los niños superdotados y con talento son aquellos que poseen, o son capaces de desarrollar, este conjunto compuesto de rasgos y aplicarlos a cualquier área potencialmente valiosa de realización humana (Ref. por Mönks y W. van Boxtel, 1992, p. 310).

Asimismo, explican que es importante señalar que ningún grupo de rasgos aislados:

Crea la sobredotación... cada grupo contribuye de igual forma, el compromiso en la tarea y la creatividad no son simplemente extras; son ingredientes igual de importantes en la composición de una persona superdotada (Renzulli y otros, ref. por Mönks y W. van Boxtel, 1992, p. 310).

\section{Teoria de los tres anillos}

Renzulli (ref. por Mönks y W. van Boxtel, 1992) representa los tres grupos gráficamente (capacidades generales por encima de la media, altos niveles de implicación en la tarea, altos niveles de creatividad), mediante tres anillos entrelazados, en los que cada grupo aporta, de igual forma, para la composición de una persona superdotada. En el Diagrama $N^{\circ} 1$ se presenta la teoría de Renzulli.

Los tres anillos de la sobredotación

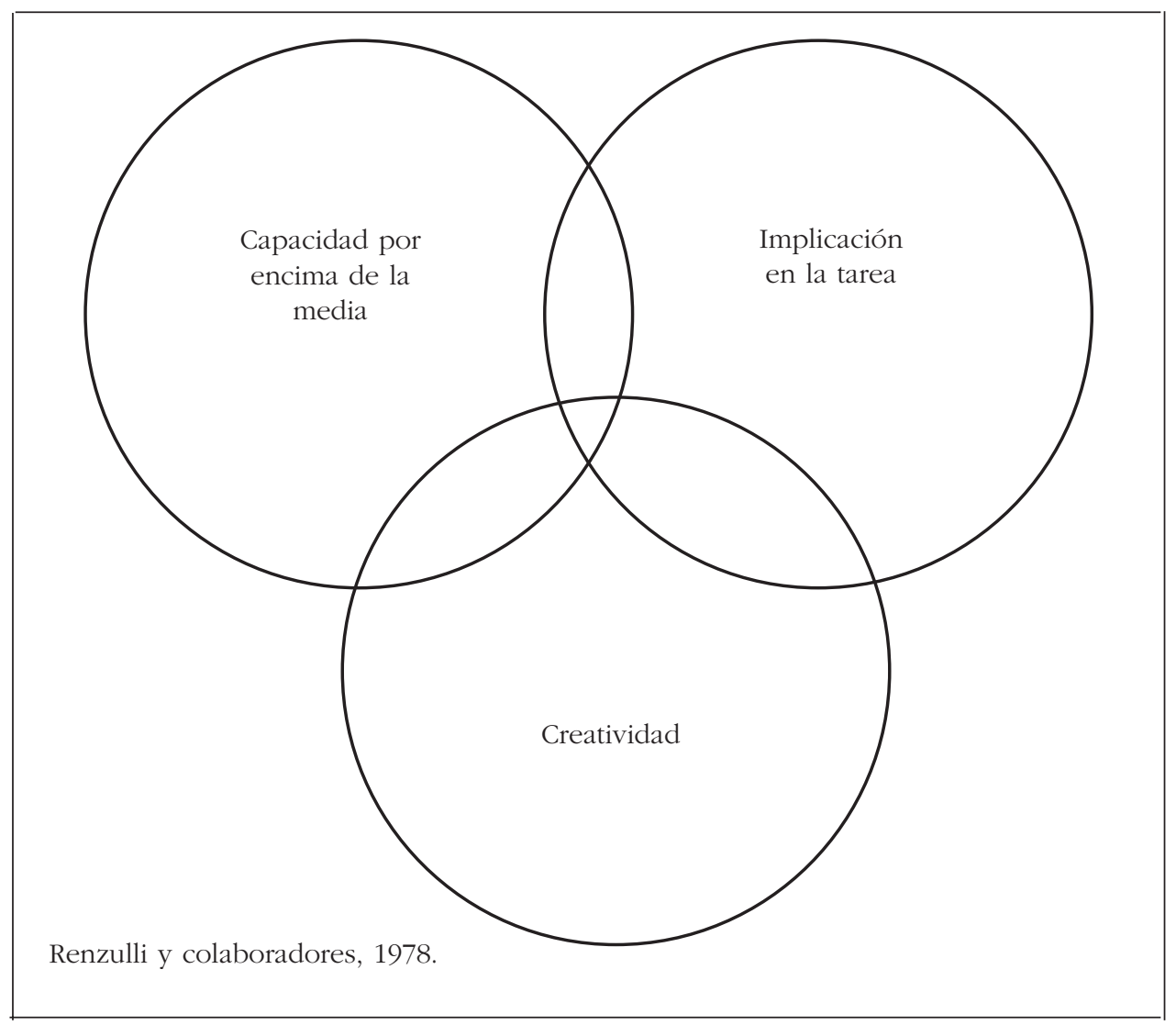

Mönks y W. van Boxtel, 1992, p. 311. 


\section{Primer grupo: la capacidad por encima de la media}

Se considera que, así como en los otros dos grupos, la capacidad por encima de la media es un constructo psicológico y que, para ser medido, debe ser operacionalizado. Con esta finalidad se realiza la aplicación de la prueba de inteligencia general o rendimiento y la de capacidades especiales.

Renzulli (1981) previene acerca de una dependencia excesiva de estos indicadores tradicionales, ya que en ellos se puede cuestionar su fiabilidad (en los intervalos extremos) y su tendencia a favorecer a los que realizan correctamente esas pruebas y a los que logran un dominio del aprendizaje. Por lo tanto, recomienda:

Evitar la utilización de puntos "separación" muy altos, para poder incluir de un $15-20$ por 100 de toda la población en estudio.

- Utilizar puntuaciones de rendimiento como:

* Calificaciones escolares

* Medias del curso o informes anecdóticos

* Ejemplos de los trabajos de los alumnos

Utilizar opiniones del profesor, de los padres de familia, de los compañeros, de uno mismo (Renzulli, ref. por Mönks y W. van Boxtel, 1992, p. 311).

\section{Segundo grupo: creatividad}

En la argumentación de Renzulli sobre la creatividad, se encuentra implícita la idea de que es una función de una serie de características en las que señala la originalidad de pensamiento, la novedad del enfoque, la capacidad para pasar por alto los convencionalismos y procedimientos establecidos y "la originalidad, la innovación o lo singular de la contribución de una persona" (Renzulli y otros, ref. por Mönks y W. van Boxtel, 1992, p. 311).

Debido a problemas que se presentan con la medición de la creatividad, Renzulli (1981) no es partidario del test de creatividad; recomienda, por lo tanto, que para realizar una mejor identificación de la creatividad de una persona, se pueden utilizar procedimientos de estimación, que evalúen sus realizaciones y productos pasados y presentes, de tal forma que se pueda estimar su potencial creativo en cada etapa de su vida.

\section{Tercer grupo: implicaciones en la tarea}

La implicación en la tarea es definida por Renzulli (1981) como una forma centrada de motivación,

considerando que la motivación se define normalmente, desde el punto de vista de un proceso estimulante general, que provoca una serie de respuestas en el organismo; la implicación en la tarea representa la energía acumulada para hacer frente a un problema particular (la tarea), de un área específica de realización (Renzulli y otros, ref. por Mönks y W. van Boxtel, 1992, p. 312).

Con el compromiso de la tarea, Renzulli menciona los factores que se encuentran asociados con ésta:

- La persistencia en la conclusión de un propósito.

- La motivación intrínseca y una fuerte absorción en el trabajo.

Independencia.

- Confianza en sí mismo e iniciativa propia.

Renzulli (1981) propone la identificación sin necesidad de medida, basándose con 
mayor énfasis en las estimaciones a partir de las ejecuciones, que en los otros dos grupos anteriores de rasgos.

El concepto de los "Tres anillos" de Renzulli, con respecto a la sobredotación, es un aporte significativo para las definiciones existentes. Su modelo expone que las características personales descritas poseen una naturaleza estática, que se evidencia mediante la referencia constante de "rasgos" (Mönks y W. van Boxtel, 1992).

Por ser la adolescencia un período evolutivo en el que se realizan procesos de transformación complejos en el marco social, en el que con frecuencia son de suma importancia para el desarrollo personal las experiencias y los procesos de socialización, en un contexto evolutivo y social, en ella deben ser ubicados los tres grupos de rasgos de Renzulli, de la sobredotación (Mönks y W. van Boxtel, 1992).

\section{Modelo triádico de la sobredotación}

El "Modelo Triádico" que presentan Mönks y W. van Boxtel (1992) es una conceptualización modificada de los "Tres Anillos" de Renzulli (1981), en la que se destaca la inclusión de los marcos sociales y su influencia en los tres rasgos: implicación en la tarea, capacidad por encima de la media y creatividad. Estos se sitúan en el período evolutivo de la adolescencia, en el que ocurren procesos de transformación complejos en el marco social, en momentos en que las experiencias y procesos de socialización son, con frecuencia, fundamentales para su desarrollo.

Mönks y W. van Boxtel (1992) sitúan la sobredotación en un contexto evolutivo y social; en él indican que los adolescentes superdotados actúan (o dejan de actuar) en una interacción dinámica constante con otras personas, que son significativas para el desarrollo de sus capacidades cognitivas.

En los aspectos evolutivos refieren el criterio de Piaget y de otros psicólogos, al señalar que en la adolescencia las capacidades generales cognitivas e intelectuales se encuentran todavía en proceso de desarrollo.

Baacke indica que

la inteligencia del adolescente no puede ser representada como una capacidad global, ya que está experimentando una diferenciación en diversos componentes, y resalta la importancia de este período para el desarrollo intelectual (ref. por Mönks y W. van Boxtel, 1992, p. 312).

Además, plantea que

la cualidad y la expresión de las capacidades del aprendizaje de los jóvenes, dependen en gran medida de factores sociales, aunque están menos afectados por las influencias ambientales de la socialización primaria, que por los incentivos y la orientación de intereses mediados por la socialización secundaria. (Ref. por Mönks y W. van Boxtel, 1992, p. 312).

La adolescencia es un período en el que se suscitan cambios psicológicos, físicos y emocionales, que son determinantes para el desarrollo integral y armónico de sus capacidades.

El adolescente talentoso, en este período crítico de la vida, se encuentra particularmente receptivo a cambios en el pensamiento creativo (Mönks y W. van Boxtel, 1992).

Dacey indica que

como la creatividad es en gran medida un asunto del concepto de uno mismo y de la motivación, y como la adolescencia es un período en el cual se define el autoconcepto, la creatividad puede ser fomentada durante este período (ref. por Mönks y W. van Boxtel, 1992, p. 313).

La implicación en la tarea es una característica de la personalidad que no es nueva en esta etapa, sino que se plantea como una forma de motivación de logro:

Es durante la adolescencia cuando es posible realizar algunos cometidos, con el conocimiento creciente de capacidades e intereses, y como el resultado de un proceso evolutivo (Mönks y W. van Boxtel, 1992, p. 113).

Estos procesos evolutivos se realizan en marcos sociales que son relevantes, debido a la interacción dinámica permanente que establece el adolescente con las personas que lo rodean. 
Diagrama $\mathrm{N}^{\circ} 2$

Modelo Triádico de la sobredotación

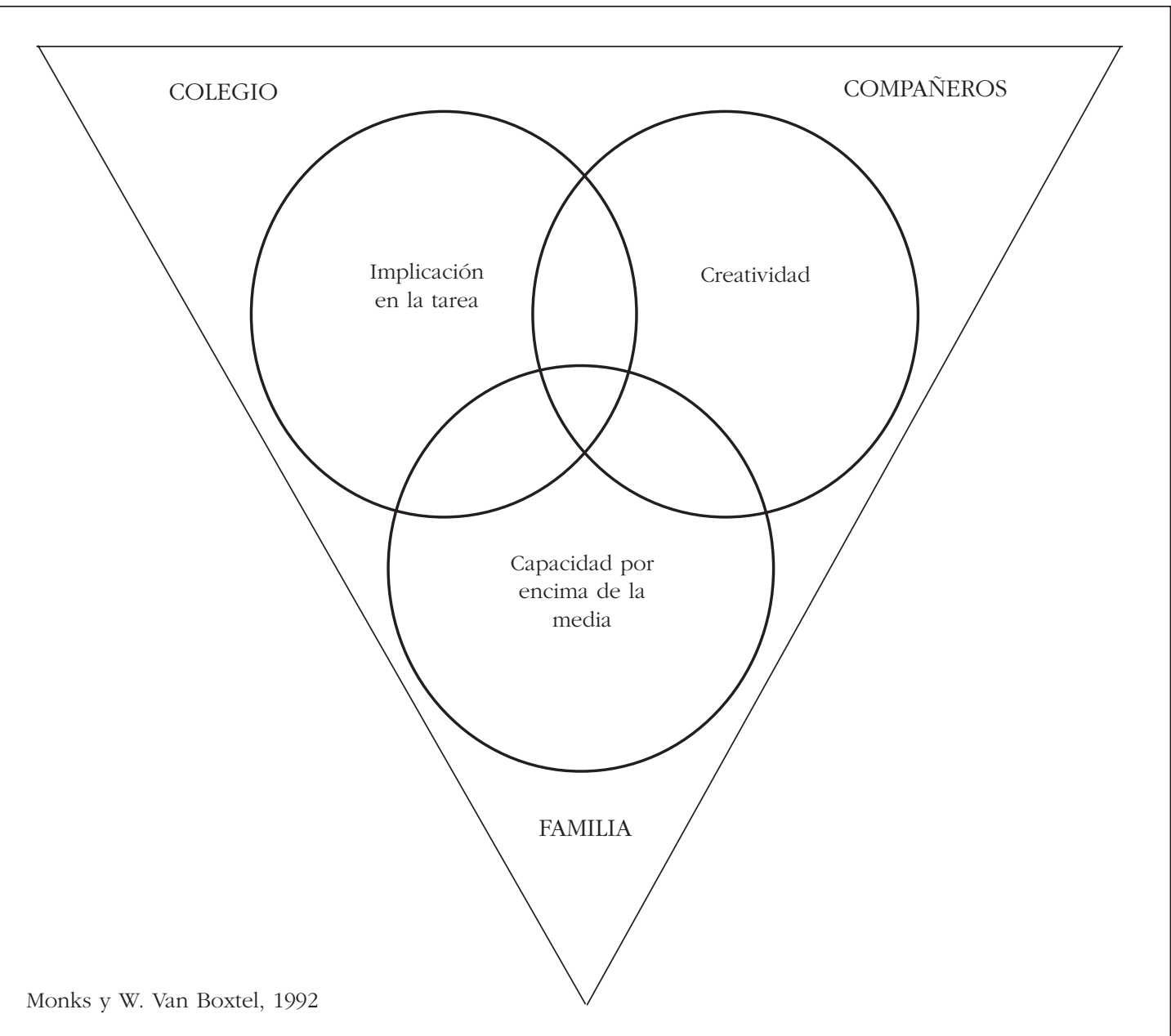

Freeman, Joan, 1992.

Mönks y W. van Boxtel (1992) denotan la importancia de la relación de la ecología social de la adolescencia, debido a que ésta estudia la interacción entre el entorno social y el comportamiento social del género humano. Refieren a Baacke (1979), quien distingue unas zonas ecológicas que son distintas en el mundo del adolescente y las establece como círculos concéntricos, en los cuales la familia, el colegio y el grupo de compañeros son reconocidos como los marcos sociales más importantes.

La familia se destaca por la seguridad y la confianza que de ella adquiere el adolescente talentoso, por medio de los lazos familiares y de las relaciones entrañables que se establecen entre todos sus miembros. 
La relevancia del interés de la familia en el proceso del desarrollo cognitivo y socioafectivo del individuo, adquiere mayor significancia en el período de la adolescencia, en el que se requiere de los padres una actitud positiva ante los cambios en las relaciones afectivas y sociales que experimentan los hijos. Es fundamental la motivación de los padres ante el esfuerzo y la dedicación que realice el adolescente talentoso, en pro del logro de sus metas; de esta interrelación positiva obtiene un nivel de satisfacción compartida en el núcleo familiar, al desarrollo de sus potencialidades.

Tabackman, en su estudio sobre los factores familiares que podrían estar relacionados con la sobredotación, indica:

Se descubrió que las familias de los niños superdotados, se encontraban significativamente por encima de la media, en relación al factor de orientación del adulto; estas familias se veían a sí mismas en sus interacciones como más independientes, permisivas, intelectuales, inestructuradas y armoniosas que la media (ref. por Mönks y W. van Boxtel, 1992, p. 314).

Asimismo, Colangelo y Deltman señalan características familiares significativas:

Por ejemplo, las familias de los chicos superdotados, tienden a mostrar fuertes lazos familiares y relaciones entrañables entre sus miembros; se observa que los padres dan a sus hijos más libertad, que están más unidos con su hijo superdotado (ref. por Mönks y W. van Boxtel, 1992, p. 322).

En estos estudios se evidencia la importancia de los ambientes familiares estimulantes para el desarrollo del talento cognitivo y de otros talentos.

En el adolescente, el desarrollo de la implicación en la tarea se encuentra influenciado por los marcos sociales, en forma especial por el colegio; sin embargo, los sistemas educativos (con muy pocas excepciones) consideran estrategias metodológicas, currículos específicos acordes con sus necesidades socioafectivas, intereses y problemas particulares de los estudiantes.

En torno a la educación de los adolescentes talentosos se plantean posiciones diversas. En la obra Estudio de la Juventud Matemáticamente Precoz, Rüpell formula las siguientes críticas.

- La sobredotación es estadísticamente definida como la capacidad eficiente para aprender en las condiciones escolares dadas.

- Los niños superdotados son identificados solo sobre la base de los tests de aptitud, sin referencia a juicios de los padres y/o profesor; de tal manera que los alumnos potencialmente superdotados puede que no sean reconocidos, y no se presta ninguna atención a los puntos fuertes y débiles de cada individuo.

- $\quad$ El niño superdotado individual es aislado y apartado de su grupo de iguales, se le sitúa en un medio de aprendizaje extraño y distinto.

- La aceleración específica de contenidos, conduce al procesamiento cada vez más y más rápido de los contenidos de aprendizaje, sin una atención explícita al desarrollo de las capacidades de pensamiento (ref. por Mönks y W. van Boxtel, 1992, p. 314).

Rüppell (ref. por Mönks y W. van Boxtel, 1992) propone el desarrollo óptimo del talento de los niños sobredotados, en un modelo de estimulación ecológicamente aceptable, el cual permite a los niños superdotados la adopción de estrategias de pensamiento completo, desde diversas condiciones estimulantes del entorno, en el contexto de su propio grupo de iguales. El desarrollo del talento cognitivo y de otros talentos, se logra por medio de la asignación de roles y de actividades específicas, dentro del mismo contexto del grupo de iguales y en relación con el desarrollo social y emocional del niño.

Al respecto, Mönks y W. van Boxtel indican: 
Las amistades tienen un significado específico cuando se definen como relaciones entre pares o iguales, siendo un par alguien igual desde el punto de vista del desarrollo, pero diferente de un compañero de la misma edad. Esta distinción es extremadamente importante para los adolescentes superdotados, ya que el desarrollo de sus capacidades sociales e intelectuales a menudo está por encima de sus compañeros de edad (ref. por Freeman, 1992, p. 308).

$\mathrm{Al}$ proveer alternativas educativas para que el adolescente talentoso se dedique a lo que es de su mayor interés personal, con su grupo de iguales, se le ofrecen oportunidades para el logro de sus metas, en un ambiente favorecedor para su desarrollo. Sin embargo, prevalece en la planificación de los programas educativos, la cientificidad, la racionalidad y la medición como ejes precursores de la formación del individuo, que limitan las posibilidades del desarrollo de sus potencialidades en otras áreas.

Freeman indica:

...la cognición ha sido teóricamente separada del afecto, en perjuicio de las materias (y niños) que requieren ambos, tales como el arte o el teatro y su actividad precursora -el juego-. Los superdotados son a menudo disuadidos de tomarse en serio esas materias de menor categoría educativa; tal como Deslike descubrió por propia experiencia y posteriormente como profesor de un colegio, porque se decía que estos niños eran demasiado listos para tales ocupaciones poco satisfactorias (Freeman, 1992, p. 308).

La educación para captar la atención de los estudiantes talentosos debe ser apropiada para llenar sus expectativas: flexible, diferenciada, actualizada, atractiva, abierta en todos los campos del saber, posibilitadora del desarrollo de las potencialidades individuales.

Mönks y W. van Boxtel indican que:

los modelos para la investigación y la práctica educativa, que toman en consideración la importancia especial del marco social,... hacen justicia a los niños superdotados como seres humanos con necesidades sociales y emocionales y son, además, más prometedores (ref. por Freeman, 1992, p. 315).

En la adolescencia se incrementa la importancia de las relaciones con los compañeros, lo cual es de igual significancia dentro o fuera del colegio; es frecuente e intensa, se establecen vínculos entre el grupo de iguales y se produce una separación creciente de la familia.

Mönks y W. van Boxtel, al referirse a las relaciones de los estudiantes talentosos, indican:

De hecho, las circunstancias excepcionales del superdotado pueden intensificar sus amistades potenciales; por ejemplo, cuando los jóvenes superdotados realizan un gran esfuerzo para desarrollar unas características personales atractivas, como la amabilidad, o el deseo demostrado de poner a disposición de sus compañeros de edad sus capacidades extra intelectuales y creativas (ref. por Freeman, 1992, p. 318).

Los marcos sociales son para el adolescente talentoso muy importantes; ocurren en ellos los procesos evolutivos de transformación sumamente complejos, en los que se destaca la interactuación constante con otros individuos significativos para ellos. El iniciar y establecer nuevos lazos afectivos es fundamental para lograr un desarrollo y equilibrio personal. De acuerdo con Mönks y W. van Boxtel, las transformaciones psicosociales se encuentran evolutivamente dentro del ciclo vital completo y la adolescencia es el período en el que se manifiestan y adquieren mayor relevancia.

En la adolescencia las relaciones afectivas adquieren relevancia, pues se incrementa el número de amistades, así como la influencia de los compañeros en su comportamiento personal. Mönks y W. van Boxtel refieren a Hartup e indican al respecto:

El afecto es una experiencia fundamental en la vida, que determina los vínculos humanos desde una temprana edad. Aunque los lazos emocionales sufren modificaciones en la mitad de la infancia, la separación aumenta durante la adolescencia, de tal manera que los amigos y otro de fuera de la familia, llegan a ser más significativos; con frecuencia es un cambio dramático y cargado de conflicto (Mönks y W. van Boxtel, 1992, p. 308).

En la adolescencia se manifiesta la importancia que tienen las relaciones interpersonales, en las que el afecto adquiere un significado especial en el desarrollo de las 
relaciones del estudiante talentoso con su grupo de iguales. En el marco social del grupo de compañeros comparte sus inquietudes, problemas, logros e ideales; proyecta sus capacidades cognitivas, extraintelectuales y su potencial creativo, en la ayuda que proporcionan a sus amistades ante alguna solicitud que éstos le presenten.

El adolescente talentoso, para el desarrollo de su potencial, requiere del apoyo y del estímulo de los familiares, de su colegio y del ambiente en que se desenvuelve. Benito, en su estudio sobre la identificación del superdotado, presenta una autobiografía en la que indica:

Que seamos superdotados no implica que hagamos genialidades o algo especial. Para que llegáramos a hacerlas, necesitábamos recibir ayuda y estímulos desde pequeños: en el colegio, en casa,... Si nadie nos echa una mano, nuestra capacidad será siempre potencial, no llegará a ser utilizada ni aflorar o reflejarse en resultados excepcionales (Benito, 1990, p. 32).

\section{Conclusiones}

Es evidente la necesidad de investigación sistemática de diversos sectores en el nivel nacional, en cuanto a factores relacionados con la educación de los niños talentosos, especialmente desde el nacimiento, que contemple aspectos del desarrollo físico, mental y social, considerando la interacción del niño, la familia y el ambiente socioeducativo.

El programa de estudio para el adolescente talentoso debe ser flexible, para que pueda adaptarse a sus altas expectativas cognitivas; diferenciado, para brindarle oportunidad de desarrollo a sus aptitudes y habilidades; incentivador de su capacidad creativa; accesible a las necesidades de interrelación con su grupo de iguales y con la proyección de su capacidad de liderazgo en el ámbito educativo.

Las experiencias en el proceso educativo son altamente significativas para el desarrollo del potencial excepcional en el aprendizaje y en el rendimiento académico elevado. Es esencial la interrelación del estudiante con el equipo de profesionales en administración y docencia de la institución, que motiven y promuevan sus expectativas y logros personales.

Al analizar el concepto de la naturaleza del talento sobresaliente en el individuo, se determina que el desarrollo de las capacidades potenciales (cognitivas y especiales) es un proceso complejo, dinámico, que implica una interrelación de factores personales, familiares, sociales y culturales en el que desarrollan y se proyectan los atributos espirituales, intelectuales, emocionales y sociales de la persona que los posee.

\section{Referencias bibliográficas}

Benito M., Yolanda. Problemática del niño superdotado. Salamanca: Ediciones Amarú. 1990.

Fonseca, Clotilde. Logo: "Un medio para el desarrollo del talento". Revista Educación. Volumen 15(1):19-30. San José, Costa Rica: Editorial de la Universidad de Costa Rica. 1991.

Freeman, Joan. Los niños superdotados. Aspectos psicológicos y pedagógicos. Trad. Illueca García, Ma. del Carmen. Madrid, España: Editorial Santillana, S.A. 1992.

Gallagher, James J. Evolución de la educación del superdotado en diferentes culturas. En Freeman, Joan. Los niños superdotados. Aspectos psicológicos y pedagógicos. Trad. Illueca García, Ma. del Carmen. Madrid. España: Editorial Santillana, S.A. 1992.

García Etchegoyhen de Lorenzo, Eloísa. El nino retardado mental y el niño superdotado talentoso: ¿Son ellos las dos caras de una misma moneda? Montevideo: Instituto Interamericano del Niño. 1985. 
López Avendaño, Olimpia. Teoría sociológica: La escuela y sus maestros. Cap. II. Documento mimeografiado. 1994.

Machado, Luis Alberto. La revolución de la inteligencia: El derecho a ser inteligente. Venezuela: Editorial Planeta Venezolano. 1983.

Maker C., June. Educación del superdotado. Tendencias significativas. Documento proyecto: Apoyo a la atención y desarrollo de las aptitudes y talentos de los niños y niñas de escuelas públicas. Ministerio de Educación Pública. 1995.

Mönks, Franz J. y W. van Boxtel, Herman. Los adolescentes superdotados: Una perspectiva evolutiva. En Freeman, Joan. Los niños superdotados. Aspectos psicológicos y pedagógicos. Trad.
Illueca García, Ma. del Carmen. Madrid, España: Editorial Santillana, S.A. 1992.

Pacheco U., Vivian Ma. Análisis cualitativo de factores que influyen en la excelencia académica de estudiantes de primeros promedios de admisión en la Universidad de Costa Rica. Tesis del Programa de Estudios de Posgrado en Educación. Universidad de Costa Rica. San José, Costa Rica. 1997.

Piaget, Jean. El nacimiento de la inteligencia en el niño. Trad. Bordonaba, Pablo. Colección Los Noventa. Editorial Grijalbo, S.A. México, 1990.

Pöggeler, Franz. "El derecho fundamental a la educación". Revista Educación. Colección. 